\title{
DISCONTINUOUS FINITE ELEMENT METHODS FOR INTERFACE PROBLEMS: ROBUST A PRIORI AND A POSTERIORI ERROR ESTIMATES*
}

\author{
ZHIQIANG $\mathrm{CAI}^{\dagger}$, CUIYU $\mathrm{HE}^{\dagger}$, AND SHUN ZHANG ${ }^{\ddagger}$
}

\begin{abstract}
For elliptic interface problems in two and three dimensions, this paper studies a priori and residual-based a posteriori error estimations for the Crouzeix-Raviart nonconforming and the discontinuous Galerkin finite element approximations. It is shown that both the a priori and the a posteriori error estimates are robust with respect to the diffusion coefficient, i.e., constants in the error bounds are independent of the jump of the diffusion coefficient. The a priori estimates are also optimal with respect to local regularity of the solution. Moreover, we obtained these estimates with no assumption on the distribution of the diffusion coefficient.
\end{abstract}

Key words. interface problem, a priori error estimation, a posteriori error estimation, discontinuous Galerkin, nonconforming

AMS subject classifications. $65 \mathrm{~N} 30,65 \mathrm{~N} 15$

DOI. $10.1137 / 16 \mathrm{M} 1056171$

1. Introduction. As a prototype of problems with interface singularities, this paper studies a priori and a posteriori error estimations of various finite element methods for the following interface problem (i.e., the diffusion problem with discontinuous coefficients):

$$
-\nabla \cdot(\alpha(x) \nabla u)=f \quad \text { in } \Omega
$$

with homogeneous Dirichlet boundary conditions (for simplicity)

$$
u=0 \quad \text { on } \partial \Omega,
$$

where $\Omega$ is a bounded polygonal domain in $\mathbb{R}^{d}$ with $d=2$ or $3 ; f \in L^{2}(\Omega)$ is a given function; and diffusion coefficient $\alpha(x)$ is positive and piecewise constant with possible large jumps across subdomain boundaries (interfaces):

$$
\alpha(x)=\alpha_{i}>0 \text { in } \Omega_{i} \text { for } i=1, \ldots, n .
$$

Here, $\left\{\Omega_{i}\right\}_{i=1}^{n}$ is a partition of the domain $\Omega$ with $\Omega_{i}$ being an open polygonal domain. The variational formulation for the interface problem in (1.1) and (1.2) is to find $u \in H_{0}^{1}(\Omega)$ such that

$$
(\alpha \nabla u, \nabla v)=(f, v) \quad \forall v \in H_{0}^{1}(\Omega) .
$$

${ }^{*}$ Received by the editors January 11, 2016; accepted for publication (in revised form) November 11, 2016; published electronically February 23, 2017.

http://www.siam.org/journals/sinum/55-1/M105617.html

Funding: The work of the first and second authors was supported in part by the National Science Foundation under grants DMS-1217081 and DMS-1522707. The work of the third author was supported in part by the Hong Kong Research Grants Council under GRF grant project 11303914, CityU 9042090.

${ }^{\dagger}$ Department of Mathematics, Purdue University, West Lafayette, IN 47907-2067 (caiz@purdue.edu, he75@purdue.edu).

${ }^{\ddagger}$ Department of Mathematics, City University of Hong Kong, Hong Kong SAR, China (shun.zhang@cityu.edu.hk). 
It is well known that the solution $u$ of problem (1.3) belongs to $H^{1+s}(\Omega)$ with possibly very small $s>0$ (see, e.g., [22]).

Let $\mathcal{T}=\{K\}$ be a regular triangulation of the domain $\Omega$ (see, e.g., [13]). Denote by $h_{K}$ the diameter of the element $K$. Assume that interfaces $\left\{\partial \Omega_{i} \cap \partial \Omega_{j}: i, j=\right.$ $1, \ldots, n\}$ do not cut through any element $K \in \mathcal{T}$. For any element $K \in \mathcal{T}$, denote by $P_{k}(K)$ the space of polynomials on $K$ with total degree less than or equal to $k$. Denote the continuous finite element space on the triangulation $\mathcal{T}$ by

$$
V_{k}=\left\{v \in H_{0}^{1}(\Omega):\left.v\right|_{K} \in P_{k}(K) \forall K \in \mathcal{T}\right\}
$$

Then the conforming finite element method is to find $u_{k}^{c} \in V_{k}$ such that

$$
\left(\alpha \nabla u_{k}^{c}, \nabla v\right)=(f, v) \quad \forall v \in V_{k} .
$$

1.1. A priori error estimate. The following a priori error estimate was established in [5]:

$$
\left\|\alpha^{1 / 2} \nabla\left(u-u_{k}^{c}\right)\right\|_{0, \Omega}=\inf _{v \in V_{k}}\left\|\alpha^{1 / 2} \nabla(u-v)\right\|_{0, \Omega} \leq C\left(\sum_{K \in \mathcal{T}} h^{2 s} \alpha_{K}\|\nabla u\|_{s, K}^{2}\right)^{1 / 2}
$$

where $\alpha_{K}=\left.\alpha\right|_{K}$ is the restriction of the $\alpha$ on element $K$. Here and thereafter, we use $\mathrm{C}$ with or without subscripts to denote a generic positive constant that is independent of the mesh parameter and the jump of $\alpha(x)$ but that may depend on the domain $\Omega$.

The estimate in (1.5) is robust with respect to $\alpha$, i.e., the constant $C$ in (1.5) is independent of $\alpha$. However, estimate (1.5) is not optimal with respect to the local regularity since $s$ is a global exponent. This kind of a priori error estimate is not satisfactory. For example, for the well-known Kellogg's example of the interface problem in $[22,9]$, the solution has low regularity at the elements attached to the origin but is very smooth away from the origin.

By Sobolev's embedding theorem (see, e.g., [20]), $H^{1+s}(\Omega)$, with $s>0$ for two dimensions and $s>1 / 2$ for three dimensions, is embedded in $C^{0}(\Omega)$ and, hence, the nodal interpolation of the solution $u$ is well-defined. In [16], it is proved that if $v \in H^{1+s}(K)$ with $s>0$ in two dimensions, then for $0<t \leq s$, the following estimate holds for the linear nodal interpolation $I_{K}$ :

$$
\left\|v-I_{K} v\right\|_{0, K} \leq C h^{1+t}|\nabla v|_{t, K}
$$

Under the local regularity assumption that the restriction of the solution $u$ of problem (1.5) on element $K$ belongs to $H^{1+s_{K}}(K)$ for all $K \in \mathcal{T}$, (1.6) implies the following a priori error estimate (see section 3.3 of [27] in two dimensions):

$$
\left\|\alpha^{1 / 2} \nabla\left(u-u_{k}^{c}\right)\right\|_{0, \Omega} \leq C\left(\sum_{K \in \mathcal{T}} h_{K}^{2 \min \left\{k, s_{K}\right\}} \alpha_{K}|\nabla u|_{s_{K}, K}^{2}\right)^{1 / 2}
$$

This estimate is not only robust with respect to the jump of $\alpha$ but also optimal with respect to the local regularity.

In three dimensions, in the case that $s>1 / 2$, using the technique of [16], one may prove the validity of estimate (1.6) that, in turn, implies the a priori error estimate in (1.7). When $s$ is only in $(0,1 / 2]$ in three dimensions, to obtain estimate (1.7) (see $[12]$ ), we need an additional assumption on the distribution of the coefficient $\alpha(x)$ (see [28] for details). 
QUASI-MONOTONICITY ASSUMPTION. Assume that any two different subdomains $\bar{\Omega}_{i}$ and $\bar{\Omega}_{j}$, which share at least one point, have a connected path passing from $\bar{\Omega}_{i}$ to $\bar{\Omega}_{j}$ through adjacent subdomains such that the diffusion coefficient $\alpha(x)$ is monotone along this path.

This assumption is very restrictive but is needed for a priori and a posteriori error estimations to be robust with respect to the diffusion coefficient $\alpha(x)$ (see, e.g., $[5,8,9,10,11,28])$. It also appeared in the convergence analysis of the domain decomposition method in [15].

The a priori error estimate using local regularity in (1.7) is the base for adaptive finite element methods to achieve equal discretization error distribution (see [26] for examples in both one and two dimensions). The first purpose of this paper is to derive a priori estimates of this type for the Crouzeix-Raviart (CR) nonconforming and the discontinuous Galerkin finite element approximations, when the solution of (1.3) has low global regularity, i.e., $s \in(0,1 / 2]$ in three dimensions, and the distribution of the coefficient does not satisfy the quasi-monotonicity assumption (QMA).

Derivation of such estimates for the nonconforming and the discontinuous elements is nontrivial. To do so, we first prove a robust Céa's lemma type of result for the CR nonconforming and the discontinuous Galerkin finite element approximations. Besides making use of both analytical approaches developed recently in [8] and [21], respectively, we also need to establish new trace inequalities (see Lemmas 2.3 and 2.4 ), which play an important role in the a posteriori error estimates as well.

Standard a priori error estimates for the nonconforming and the discontinuous elements (see, e.g., $[3,30]$ ) require the underlying problem to be sufficiently smooth, i.e., at least piecewise $H^{3 / 2+\epsilon}$, so that there is an error equation. For problems with low regularity, by carefully defining duality pairs on element interfaces, in [8] we developed a nonstandard variational formulation that, in turn, leads to an error equation and then an a priori error estimate. The estimate in [8] is robust with respect to $\alpha$ without the QMA but not locally optimal due to the use of a continuous approximation in our analysis. An alternative approach was developed by Gudi [21] for the Poisson equation. His approach compares the discontinuous solution with the continuous solution and makes use of the efficiency bound of the a posteriori error estimation. Moreover, it is applicable to problems with low regularity. Its application to interface problems with the Oswald interpolation analyzed in [8] would yield an a priori error estimate that is robust under the QMA.

1.2. A posteriori error estimate. For the conforming finite element approximation in (1.4), by using the diffusion coefficient to properly weight the element residual and the edge flux jump, Bernardi and Verfürth in [5] (see also [28]) showed that the resulting residual based error estimator is locally efficient and globally reliable with the efficiency constant independent of the jump of the diffusion coefficient. Moreover, under the QMA, the reliability constant is proved to be uniform with respect to the jump as well. Since then, various robust a posteriori error estimators have been constructed, analyzed, and implemented (see, e.g., $[24,9,31]$ for the conforming elements, $[1,10]$ for the nonconforming elements, $[2,23,10,14]$ for the mixed elements, and $[8,18,19]$ for the discontinuous elements). The robustness for those estimators was theoretically established again under the QMA. However, numerical results by many researchers including ours strongly suggest that those estimators are robust even when the diffusion coefficients are not quasi-monotone.

The second purpose of this paper is to theoretically establish robust reliability bounds of the residual estimators without the QMA for the nonconforming and 
the discontinuous elements. The QMA is imposed to guarantee the desired approximation and stability properties of the Clément type interpolation (see [5] for details), which is one of the key steps in obtaining the reliability bound of the residual based estimator. For the conforming elements, this type of interpolation is defined on vertex patches through averaging and, hence, the QMA is required. For the nonconforming and the discontinuous elements, one may construct a modified Clément interpolation satisfying the desired properties without the QMA (see [7]). Due to the lack of the error equation, the reliability bound for discontinuous approximations is commonly analyzed through the Helmholtz decomposition of the true error. Application of the Helmholtz decomposition, in turn, leads to establishment of the reliability bound for conforming approximations and, hence, the requirement of the QMA.

In [7], we introduced a new and direct analysis that does not involve the Helmholtz decomposition for two-dimensional nonconforming elements. In particular, we derived an $L^{2}$ representation of the error in the energy norm that naturally contains three terms: the element residual, the face flux jump, and the face solution jump. Due to a technical difficulty, the solution jump was modified at elements where the QMA is not satisfied. The modified estimator was proved to be robustly reliable without the QMA. Unfortunately, robustness of local efficiency of the modified indicator requires the QMA.

With the help of our newly developed trace inequality (Lemmas 2.3 and 2.4), we are able to bound the solution jump without any modification. Moreover, instead of using the nonconforming Clément type interpolation as in [7], we use the standard nonconforming interpolation and the piecewise constant projection for the respective nonconforming and discontinuous elements, which fully takes advantage of the local feature of the element itself. Both the approximations are elementwisely defined. Thus, without the QMA, we are able to prove the robustness in both two and three dimensions for the CR nonconforming and the discontinuous elements.

The paper is organized as follows. Section 2 introduces Sobolev spaces of fractional order and establishes some new trace inequalities that play an important role in both the a priori and a posteriori error estimates. The a priori and a posteriori error estimates for the nonconforming and discontinuous Galerkin finite element methods are derived in sections 3 and 4 , respectively.

2. Sobolev space and preliminaries. This section introduces Sobolev space of fractional order and establishes new trace inequalities.

2.1. Sobolev space of fractional order. Let $\Omega$ be a nonempty open set in $\mathbb{R}^{d}$. We use the standard notation and definitions for the Sobolev spaces $H^{m}(\Omega)^{d}$ and $H^{m}(\partial \Omega)^{d}$ with integer $m \geq 0$; the standard associated inner products are denoted by $(\cdot, \cdot)_{m, \Omega}$ and $(\cdot, \cdot)_{m, \partial \Omega}$, and their respective norms (seminorms) are denoted by $\|\cdot\|_{m, \Omega}$ and $\|\cdot\|_{m, \partial \Omega}\left(|\cdot|_{m, \Omega}\right.$ and $\left.|\cdot|_{m, \partial \Omega}\right)$. We suppress the superscript $d$ because their dependence on dimension will be clear by context. We also omit the subscript $\Omega$ from the inner product and norm designations when there is no risk of confusion. For $m=0, H^{m}(\Omega)^{d}$ coincides with $L^{2}(\Omega)^{d}$. In this case, the inner product and norm will be denoted by $\|\cdot\|$ and $(\cdot, \cdot)$, respectively.

For $t \in(0,1)$, the seminorm

$$
|v|_{t, \Omega}=\left(\int_{\Omega} \int_{\Omega} \frac{|v(x)-v(y)|^{2}}{|x-y|^{d+2 t}} d x d y\right)^{1 / 2}, \quad 0<t<1,
$$


is used to define Sobolev spaces of fractional order. For integer $m \geq 0$, Sobolev space $H^{s}(\Omega)$ with $s=m+t$ is equipped with the norm

$$
\|v\|_{s, \Omega}=\left(\sum_{|\alpha| \leq m} \int_{\Omega}\left|\partial^{\alpha} v\right|^{2} d x+|v|_{s, \Omega}^{2}\right)^{1 / 2}
$$

where $|v|_{s, \Omega}$ is a seminorm defined by

$$
|v|_{s, \Omega}=\left(\sum_{|\alpha|=m}\left|\partial^{\alpha} v\right|_{t, \Omega}^{2}\right)^{1 / 2}
$$

Sobolev spaces with negative indices are defined through duality.

Another way to define Sobolev spaces of fractional order is by the method of interpolation. To this end, let $B_{1} \subset B_{0}$ be Banach spaces. For $t>0$ and $u \in B_{0}$, define the $K$-functional by

$$
K(t, u)=\inf _{v \in B_{1}}\left(\|u-v\|_{B_{0}}^{2}+t^{2}\|v\|_{B_{1}}^{2}\right)^{1 / 2} .
$$

For $0<\theta<1$, the interpolation space $B_{\theta}=\left[B_{0}, B_{1}\right]_{\theta}$ is a Banach space equipped with the norm

$$
\|u\|_{\left[B_{0}, B_{1}\right]_{\theta}}=N_{\theta}\left(\int_{0}^{\infty}\left|t^{-\theta} K(t, u)\right|^{2} \frac{d t}{t}\right)^{1 / 2}
$$

where $N_{\theta}>0$ is a normalization factor.

For any real numbers $s_{0} \leq s_{1}$, let $s=m+t=(1-\theta) s_{0}+\theta s_{1}$ with $\theta \in(0,1)$. It was shown (see Theorem B.8 in [25]) that

$$
\left[H^{s_{0}}(\Omega), H^{s_{1}}(\Omega)\right]_{\theta}=H^{s}(\Omega)
$$

and that the norms defined in (2.1) and (2.3) are identical if the normalization factor is chosen to be $N_{\theta}=\sqrt{\frac{2 \sin (\pi \theta)}{\pi}}$. Moreover, for $v \in H^{s_{1}}(\Omega)$, it was shown (see Theorem B.1 in [25]) that

$$
\|v\|_{s, \Omega} \leq \sqrt{\frac{\sin (\pi \theta)}{\pi \theta(1-\theta)}}\|v\|_{s_{0}, \Omega}^{1-\theta}\|v\|_{s_{1}, \Omega}^{\theta} .
$$

Lemma 2.1. Let $s>0, t \in[0, s)$, and $K \in \mathcal{T}$. Assume that $v$ is a given function in $H^{s}(K)$. For any given $\epsilon>0$, there exists a small $\delta \in(0, s-t)$, depending on $v$, such that

$$
\|v\|_{t+\delta, K} \leq(1+\epsilon)\|v\|_{t, K} .
$$

Proof. Obviously, (2.5) holds for $v=0$. Assume that $v \neq 0$. For any $\delta \in(0, s-t)$, we have

$$
H^{t+\delta}(K)=\left[H^{t}(K), H^{s}(K)\right]_{\theta} \quad \text { with } \theta=\frac{\delta}{s-t},
$$

which, together with (2.4), implies

$$
\|v\|_{t+\delta, K} \leq \sqrt{\frac{\sin (\pi \theta)}{\pi \theta(1-\theta)}}\|v\|_{t, K}^{1-\theta}\|v\|_{s, K}^{\theta}=\sqrt{\frac{\sin (\pi \theta)}{\pi \theta(1-\theta)}}\left(\frac{\|v\|_{s, K}}{\|v\|_{t, K}}\right)^{\theta}\|v\|_{t, K} .
$$


Now, (2.5) is a consequence of the fact that

$$
\lim _{\theta \rightarrow 0} \sqrt{\frac{\sin (\pi \theta)}{\pi \theta(1-\theta)}}\left(\frac{\|v\|_{s, K}}{\|v\|_{t, K}}\right)^{\theta}=1 .
$$

This completes the proof of the lemma.

Remark 2.2. Since $\|v\|_{t, K} \leq\|v\|_{t+\delta, K}$, Lemma 2.1 implies that

$$
\lim _{\delta \rightarrow 0^{+}}\|v\|_{t+\delta}=\|v\|_{t} .
$$

Note that this continuity is not uniform with respect to $v$.

2.2. Trace inequalities. For any $K \in \mathcal{T}$ and some $\alpha>0$, let

$$
V^{1+\alpha}(K)=\left\{v \in H^{1+\alpha}(K): \Delta v \in L^{2}(K)\right\} .
$$

Lemma 2.3. Let $F$ be a face of $K \in \mathcal{T}$ and let $s>0$. Assume that $v$ is a given function in $V^{1+s}(K)$. Then there exists a small $0<\delta<\min \{s, 1 / 2\}$, depending on $v$, and a positive constant $C$ independent of $\delta$ such that

$$
\|\nabla v \cdot \mathbf{n}\|_{\delta-1 / 2, F} \leq C\left(\|\nabla v\|_{0, K}+h_{K}\|\Delta v\|_{0, K}\right) .
$$

Proof. For any $v \in V^{1+s}(K)$, it was shown in $[4,8]$ that for all $0<\delta<$ $\min \{s, 1 / 2\}$, we have

$$
\|\nabla v \cdot \mathbf{n}\|_{\delta-1 / 2, F} \leq C\left(\|\nabla v\|_{\delta, K}+h_{K}^{1-\delta}\|\Delta v\|_{0, K}\right),
$$

which, together with Lemma 2.1 with $t=0$ and the fact that $h_{K}^{-\delta} \leq 2$ for sufficiently small $\delta$, implies the validity of (2.6). This completes the proof of the lemma.

Lemma 2.4. Let $F$ be a face of $K \in \mathcal{T}, \mathbf{n}_{F}$ the unit vector normal to $F$, and $s>0$. Assume that $v$ is a given function in $V^{1+s}(K)$. For any $w_{h} \in P_{k}(K)$, we have

$$
\begin{aligned}
\int_{F}\left(\nabla v \cdot \mathbf{n}_{F}\right) w_{h} d s & \leq C h_{F}^{-1 / 2}\left\|w_{h}\right\|_{0, F}\left(\|\nabla v\|_{0, K}+h_{K}\|\Delta v\|_{0, K}\right) \\
& \leq C h_{K}^{-1}\left\|w_{h}\right\|_{0, K}\left(\|\nabla v\|_{0, K}+h_{K}\|\Delta v\|_{0, K}\right) .
\end{aligned}
$$

Proof. The second inequality in (2.7) follows from the inverse inequality. To show the validity of the first inequality in (2.7), as discussed in [8], $\int_{F}\left(\nabla v \cdot \mathbf{n}_{F}\right) w_{h} d s$ may be viewed as a duality pairing between $H^{\delta-1 / 2}(F)$ and $H^{1 / 2-\delta}(F)$ for all $0<\delta<$ $\min \{s, 1 / 2\}$. It follows from the definition of the dual norm, the inverse inequality, and (2.6) for sufficiently small $\delta$ that

$$
\begin{aligned}
\int_{F}\left(\nabla v \cdot \mathbf{n}_{F}\right) w_{h} d s & \leq\|\nabla v \cdot \mathbf{n}\|_{\delta-1 / 2, F}\left\|w_{h}\right\|_{1 / 2-\delta, F} \\
& \leq C h_{K}^{\delta-1 / 2}\left\|w_{h}\right\|_{0, F}\left(\|\nabla v\|_{0, K}+h_{K}\|\Delta v\|_{0, K}\right) \\
& \leq C h_{K}^{-1 / 2}\left\|w_{h}\right\|_{0, F}\left(\|\nabla v\|_{0, K}+h_{K}\|\Delta v\|_{0, K}\right) .
\end{aligned}
$$

This completes the proof of the first inequality in (2.7) and, hence, the lemma.

Remark 2.5. Generalizations of the above results to $\boldsymbol{\tau} \in\left\{\boldsymbol{\tau} \in H^{\alpha}(K)^{d}: \nabla \cdot \boldsymbol{\tau} \in\right.$ $\left.L^{2}(K)\right\}$ are obvious. 
3. Nonconforming finite element method. This section establishes the a priori and the residual based a posteriori error estimates for the CR nonconforming finite element approximation. To this end, let $\mathcal{N}$ be the set of vertices of the triangulation $\mathcal{T}$ and $\mathcal{N}_{D}$ be the collection of the vertices on the Dirichlet boundary. Denote by $\mathcal{E}_{K}$ the set of faces of element $K \in \mathcal{T}$. In this paper, face means edge/face in two/three dimensions. Denote the set of all faces of the triangulation $\mathcal{T}$ by

$$
\mathcal{E}:=\mathcal{E}_{I} \cup \mathcal{E}_{D}
$$

where $\mathcal{E}_{I}$ and $\mathcal{E}_{D}$ are the respective sets of all interior and boundary faces. For each $F \in \mathcal{E}$, denote by $h_{F}$ the diameter of the face $F$ and by $\mathbf{n}_{F}$ a unit vector normal to $F$. For each interior face $F \in \mathcal{E}_{I}$, let $K_{F}^{-}$and $K_{F}^{+}$be the two elements sharing the common face $F$ such that the unit outward normal vector of $K_{F}^{-}$coincides with $\mathbf{n}_{F}$. When $F \in \mathcal{E}_{D}, \mathbf{n}_{F}$ is the unit outward normal vector of $\partial \Omega$ and denote the element by $K_{F}^{-}$. For any $F \in \mathcal{E}$, denote by $\left.v\right|_{F} ^{-}$and $\left.v\right|_{F} ^{+}$, respectively, the traces of a function $v$ over $F$. Define jumps over faces by

$$
\llbracket v \rrbracket_{F}:= \begin{cases}\left.v\right|_{F} ^{-}-\left.v\right|_{F} ^{+}, & F \in \mathcal{E}_{I}, \\ \left.v\right|_{F} ^{-}, & F \in \mathcal{E}_{D} .\end{cases}
$$

Denote the CR linear nonconforming finite element space by

$$
V^{c r}=\left\{v \in L^{2}(\Omega):\left.v\right|_{K} \in P_{1}(K) \forall K \in \mathcal{T}, \int_{F} \llbracket v \rrbracket d s=0 \forall F \in \mathcal{E}\right\} .
$$

Then the nonconforming finite element method is to find $u^{c r} \in V^{c r}$ such that

$$
\left(\alpha \nabla_{h} u^{c r}, \nabla_{h} v\right)=(f, v) \quad \forall v \in V^{c r},
$$

where $\nabla_{h}$ is the discrete gradient operator defined elementwisely.

3.1. A priori error estimate. Let

$$
\begin{aligned}
W^{1,1}(\mathcal{T}) & =\left\{v \in L^{2}(\Omega):\left.v\right|_{K} \in W^{1,1}(K) \forall K \in \mathcal{T}\right\} \\
\text { and } \quad W(\mathcal{T}) & =\left\{v \in W^{1,1}(\mathcal{T}): \int_{F} \llbracket v \rrbracket d s=0 \forall F \in \mathcal{E}\right\} .
\end{aligned}
$$

Denote by $\theta_{F}(\mathbf{x})$ the nodal basis function of $V^{c r}$ associated with the face $F \in \mathcal{E}$, i.e.,

$$
\frac{1}{\left|F^{\prime}\right|} \int_{F^{\prime}} \theta_{F}(\mathbf{x}) d s=\delta_{F F^{\prime}} \forall F^{\prime} \in \mathcal{E},
$$

where $\delta_{F F^{\prime}}$ is the Kronecker delta. The local and global CR interpolants are defined respectively by

$$
I_{K}^{c r} v=\sum_{F \in \mathcal{E}_{K}}\left(\frac{1}{|F|} \int_{F} v d s\right) \theta_{F}(\mathbf{x}) \quad \text { and } \quad I^{c r} v=\sum_{F \in \mathcal{E}}\left(\frac{1}{|F|} \int_{F} v d s\right) \theta_{F}(\mathbf{x})
$$

for the respective $v \in W^{1,1}(K)$ and $v \in W(\mathcal{T})$. It was shown (see, e.g., Theorem 1.103 and Example 1.106(ii) of [17]) that for $v \in H^{1+t}(K)$ with $0 \leq t \leq 1$

$$
\left\|v-I_{K}^{c r} v\right\|_{0, K}+h_{K}\left\|\nabla\left(v-I_{K}^{c r} v\right)\right\|_{0, K} \leq C h_{K}^{1+t}|\nabla v|_{t, K} .
$$


Let $f_{0}$ be piecewise constant such that its restriction on $K \in \mathcal{T}$ is the average of $f$ on $K$. Define local and global weighted oscillations by

$$
\operatorname{osc}_{1, \alpha}(f, K)=\frac{h_{K}}{\sqrt{\alpha_{K}}}\left\|f-f_{0}\right\|_{0, K} \quad \text { and } \quad \operatorname{osc}_{1, \alpha}(f, \mathcal{T})=\left(\sum_{K \in \mathcal{T}} \operatorname{osc}_{1, \alpha}(f, K)^{2}\right)^{1 / 2}
$$

respectively.

THEOREM 3.1. Let $u$ be the solution of (1.3) and $u_{K}$ be its restriction on $K \in \mathcal{T}$. Assume that $u \in H^{1+s}(\Omega) \cap V^{1+s}(\mathcal{T})$ for some $s>0$ and that $\left.u\right|_{K} \in H^{1+s_{K}}(K)$ with elementwise defined $s_{K}>0$ for all $K \in \mathcal{T}$. Let $u^{\text {cr }} \in V^{\text {cr }}$ be the nonconforming finite element approximation in (3.1). For both two and three dimensions, the error estimates

$$
\begin{aligned}
\left\|\alpha^{1 / 2} \nabla_{h}\left(u-u^{c r}\right)\right\|_{0} & \leq C\left(\inf _{v \in V^{c r}}\left\|\alpha^{1 / 2} \nabla_{h}(u-v)\right\|_{0}+\operatorname{osc}_{1, \alpha}(f, \mathcal{T})\right) \\
& \leq C\left(\left(\sum_{K \in \mathcal{T}} h_{K}^{2 \min \left\{1, s_{K}\right\}}\left|\alpha^{1 / 2} \nabla u\right|_{s_{K}, K}^{2}\right)^{1 / 2}+\operatorname{osc}_{1, \alpha}(f, \mathcal{T})\right)
\end{aligned}
$$

hold, where $C$ is a positive constant independent of the jump of the diffusion coeffcient $\alpha$.

Proof. The second inequality in (3.3) is an immediate consequence of the first inequality in (3.3) and the approximation property in (3.2). By Strang's lemma, to show the validity of the first inequality in (3.3), it suffices to prove

$$
\sup _{w \in V^{c r}} \frac{\left|(f, w)-\left(\alpha \nabla u, \nabla_{h} w\right)\right|}{\left\|a^{1 / 2} \nabla_{h} w\right\|_{0, \Omega}} \leq C\left(\inf _{v \in V^{c r}}\left\|a^{1 / 2} \nabla_{h}(u-v)\right\|_{0, \Omega}+\operatorname{osc}_{1, \alpha}(f, \mathcal{T})\right) .
$$

To this end, for any $w \in V^{c r}$ and any $F \in \mathcal{E}$, by the fact that $\int_{F} \llbracket w \rrbracket d s=0$, the mean value of $w$ over $F$ is single-valued constant, i.e.,

$$
\bar{w}_{F}=\left.\frac{1}{|F|} \int_{F} w\right|_{K_{F}^{+}} d s=\left.\frac{1}{|F|} \int_{F} w\right|_{K_{F}^{-}} d s
$$

where $K_{F}^{+}$and $K_{F}^{-}$are two elements sharing the common face $F$. Moreover, $\bar{w}_{F}=0$ for $F \in \mathcal{E}_{D}$. Hence, by the continuity of the flux $\mathbf{n} \cdot \alpha \nabla u$ across face $F \in \mathcal{E}_{I}$, we have

$$
\sum_{K \in \mathcal{T}} \sum_{F \in \partial K} \int_{F}(\mathbf{n} \cdot \alpha \nabla u) \bar{w}_{F} d s=\sum_{F \in \mathcal{E}} \int_{F} \llbracket(\mathbf{n} \cdot a \nabla u) \bar{w}_{F} \rrbracket d s=0 .
$$

Now, it follows from (3.1), integration by parts, (3.5), the fact that $\left(\left.\mathbf{n}_{F} \cdot \alpha \nabla v\right|_{K}\right)_{F}$ is a constant, and (2.7) that for all $v \in V^{c r}$

$$
\begin{aligned}
& \left(\alpha \nabla u, \nabla_{h} w\right)-(f, w) \\
= & \sum_{K \in \mathcal{T}} \int_{\partial K}(\mathbf{n} \cdot \alpha \nabla u) w d s=\sum_{K \in \mathcal{T}} \sum_{F \in \partial K} \int_{F}(\mathbf{n} \cdot \alpha \nabla u)\left(w-\bar{w}_{F}\right) d s \\
= & \sum_{K \in \mathcal{T}} \sum_{F \in \partial K} \int_{F}(\mathbf{n} \cdot \alpha \nabla(u-v))\left(w-\bar{w}_{F}\right) d s
\end{aligned}
$$




$$
\begin{aligned}
& \leq C \sum_{K \in \mathcal{T}} \sum_{F \in \partial K} h_{K}^{-1 / 2}\left\|w-\bar{w}_{F}\right\|_{0, F}\left(\|\alpha \nabla(u-v)\|_{0, K}+h_{K}\|f\|_{0, K}\right) \\
& \leq C \sum_{K \in \mathcal{T}} \sum_{F \in \partial K}\left(\left\|\alpha^{1 / 2} \nabla(u-v)\right\|_{0, K}+h_{K} \alpha_{K}^{-1 / 2}\|f\|_{0, K}\right)\left\|\alpha^{1 / 2} \nabla w\right\|_{0, K} .
\end{aligned}
$$

The last inequality is due to the fact that $\left\|w-\bar{w}_{F}\right\|_{0, F} \leq C h_{K}^{1 / 2}\|\nabla w\|_{0, K}$. Now, the Cauchy-Schwarz inequality gives

$$
\frac{\left|\left(\alpha \nabla u, \nabla_{h} w\right)-(f, w)\right|}{\left\|a^{1 / 2} \nabla_{h} w\right\|_{0, \Omega}} \leq C\left(\inf _{v \in V^{c r}}\left\|\alpha^{1 / 2} \nabla_{h}(u-v)\right\|_{0}+\left(\sum_{K \in \mathcal{T}} h_{K}^{2} \alpha_{K}^{-1}\|f\|_{0, K}^{2}\right)^{1 / 2}\right)
$$

for all $w \in V^{c r}$. Without the QMA, we have the efficiency bound (see (3.11))

$$
h_{K} \alpha_{K}^{-1 / 2}\|f\|_{0, K} \leq C\left(\left\|\alpha^{1 / 2} \nabla_{h}(u-v)\right\|_{0, \Delta_{K}}+\operatorname{osc}_{1, \alpha}\left(f, \Delta_{K}\right)\right)
$$

for all $v \in V^{c r}$ and all $K \in \mathcal{T}$, where $\Delta_{K}$ is a local patch of elements containing $K$. Combining the above two inequalities implies the validity of (3.4). This completes the proof of the theorem.

Since the linear conforming finite element solution $u_{1}^{c}$ belongs to $V^{c r}$, we have

$$
\inf _{v \in V^{c r}}\left\|\alpha^{1 / 2} \nabla_{h}(u-v)\right\|_{0} \leq\left\|\alpha^{1 / 2} \nabla_{h}\left(u-u_{1}^{c}\right)\right\|_{0, \Omega},
$$

which, together with Theorem 3.1, implies the following robust comparison result between the linear conforming finite element and the CR nonconforming finite element approximations.

Corollary 3.2. Without the QMA, there exists a positive constant $C$ independent of the jump of the diffusion coefficient such that

$$
\left\|\alpha^{1 / 2} \nabla_{h}\left(u-u^{c r}\right)\right\|_{0, \Omega} \leq C\left(\left\|\alpha^{1 / 2} \nabla_{h}\left(u-u_{1}^{c}\right)\right\|_{0, \Omega}+\operatorname{osc}_{1, \alpha}(f, \mathcal{T})\right) .
$$

3.2. Residual-based a posteriori error estimator. This section first describes local indicator and global estimator for the CR nonconforming finite element approximation. The estimator for the nonconforming elements introduced in [7] is more accurate than the existing estimators (see, e.g., $[1,8]$ ) and differs in replacing the face tangential derivative jumps by the face solution jumps. The local indicator and the global estimator are then shown to be efficient and reliable, respectively.

3.2.1. Error indicator and estimator. For each $F \in \mathcal{E}$, let $\mathbf{t}_{F}$ be the unit vector tangent to $F$ for $d=2$, and let $\mathbf{n}_{F}$ be the unit vector normal to $F$. Denote a tangential component of a vector field $\boldsymbol{\tau}$ on $F$ by

$$
\gamma_{F}(\boldsymbol{\tau}):= \begin{cases}\boldsymbol{\tau} \cdot \mathbf{t}_{F}, & d=2 \\ \boldsymbol{\tau} \times \mathbf{n}_{F}, & d=3 .\end{cases}
$$

To this end, let $w_{F}^{+}$and $w_{F}^{-}$be weights defined on $F$ satisfying $w_{F}^{+}(x)+w_{F}^{-}(x)=1$, and introduce the following weighted averages:

$$
\{v(x)\}_{w}^{F}=\left\{\begin{array}{ll}
w_{F}^{-} v_{F}^{-}+w_{F}^{+} v_{F}^{+}, & F \in \mathcal{E}_{I}, \\
\left.v\right|_{F} ^{-}, & F \in \mathcal{E}_{D}
\end{array} \text { and }\{v(x)\}_{F}^{w}= \begin{cases}w_{F}^{+} v_{F}^{-}+w_{F}^{-} v_{F}^{+}, & F \in \mathcal{E}_{I}, \\
0, & F \in \mathcal{E}_{D}\end{cases}\right.
$$


for all $F \in \mathcal{E}$. A simple calculation leads to the following identity:

$$
\llbracket u v \rrbracket_{F}=\{v\}_{F}^{w} \llbracket u \rrbracket_{F}+\{u\}_{w}^{F} \llbracket v \rrbracket_{F} .
$$

For any $F \in \mathcal{E}_{I}$, denote by $\alpha_{F}^{+}$and $\alpha_{F}^{-}$the diffusion coefficients on $K_{F}^{+}$and $K_{F}^{-}$, respectively. Denote the arithmetic and the harmonic averages of $\alpha$ on $F \in \mathcal{E}$ by

$$
\alpha_{F, A}=\left\{\begin{array}{cl}
\frac{\alpha_{F}^{+}+\alpha_{F}^{-}}{2}, & F \in \mathcal{E}_{I}, \\
\alpha_{F}^{-}, & F \in \mathcal{E}_{D}
\end{array} \quad \text { and } \quad \alpha_{F, H}=\left\{\begin{array}{cl}
\frac{2 \alpha_{F}^{+} \alpha_{F}^{-}}{\alpha_{F}^{+}+\alpha_{F}^{-}}, & F \in \mathcal{E}_{I}, \\
\alpha_{F}^{-}, & F \in \mathcal{E}_{D},
\end{array}\right.\right.
$$

respectively, which are equivalent to the respective maximum and minimum of $\alpha$ :

$\frac{1}{2} \max \left\{\alpha_{F}^{+}, \alpha_{F}^{-}\right\} \leq \alpha_{F, A} \leq \max \left\{\alpha_{F}^{+}, \alpha_{F}^{-}\right\}$and $\min \left\{\alpha_{F}^{+}, \alpha_{F}^{-}\right\} \leq \alpha_{F, H} \leq \frac{1}{2} \min \left\{\alpha_{F}^{+}, \alpha_{F}^{-}\right\}$.

In order to guarantee the robustness of the error estimate with respect to $\alpha$, we choose harmonic weights in this paper:

$$
w_{F}^{ \pm}=\frac{\alpha_{F}^{\mp}}{\alpha_{F}^{-}+\alpha_{F}^{+}} .
$$

It is easy to show that

$$
w_{F}^{ \pm} \alpha_{F}^{ \pm} \leq \sqrt{\alpha^{ \pm} \alpha_{F, H}}, \quad \frac{\omega_{F}^{+}}{\sqrt{\alpha_{F}^{-}}} \leq \sqrt{\frac{1}{\alpha_{F, A}}}, \quad \text { and } \quad \frac{\omega_{F}^{-}}{\sqrt{\alpha_{F}^{+}}} \leq \sqrt{\frac{1}{\alpha_{F, A}}} .
$$

Denote the element residuals and the corresponding indicators by

$$
r_{K}^{c r}=\left.f_{0}\right|_{K} \quad \text { and } \quad \eta_{r, K}^{c r}=\frac{h_{K}}{\sqrt{\alpha_{K}}}\left\|r_{K}^{c r}\right\|_{0, K} \quad \forall K \in \mathcal{T},
$$

respectively. Denote the respective face flux and tangential derivative jumps by

$$
j_{n, F}^{c r}=\llbracket \alpha \nabla_{h} u^{c r} \cdot \mathbf{n} \rrbracket_{F} \quad \forall F \in \mathcal{E}_{I} \quad \text { and } \quad j_{t, F}^{c r}=\llbracket \gamma_{F}\left(\nabla_{h} u^{c r}\right) \rrbracket_{F} \quad \forall F \in \mathcal{E}
$$

and the indicators corresponding to the face flux, tangential derivative, and solution jumps by

$$
\begin{aligned}
& \eta_{j, n, F}^{c r}=\sqrt{\frac{h_{F}}{\alpha_{F}, A}}\left\|j_{n, F}^{c r}\right\|_{0, F}, \quad \eta_{j, t, F}^{c r}=\sqrt{\alpha_{F, H} h_{F}}\left\|j_{t, F}^{c r}\right\|_{0, F}, \\
& \text { and } \quad \eta_{j, u, F}^{c r}=\sqrt{\frac{\alpha_{F, H}}{h_{F}}}\left\|\llbracket u^{c r} \rrbracket\right\|_{0, F},
\end{aligned}
$$

respectively, where $h_{F}$ is the diameter of the face $F$. Then the local indicator of the residual type for the nonconforming elements, introduced in [7] and to be studied in this paper, is given by

$$
\eta_{K}^{c r}=\left(\left(\eta_{r, K}^{c r}\right)^{2}+\sum_{F \in \mathcal{E}_{K} \cap \mathcal{E}_{I}} \frac{1}{2}\left(\eta_{j, n, F}^{c r}\right)^{2}+\sum_{F \in \mathcal{E}_{K} \cap \mathcal{E}_{I}} \frac{1}{2}\left(\eta_{j, u, F}^{c r}\right)^{2}+\sum_{F \in \mathcal{E}_{K} \cap \mathcal{E}_{D}}\left(\eta_{j, u, F}^{c r}\right)^{2}\right)^{1 / 2} .
$$

Now the global estimator for the nonconforming elements is given by

$$
\eta_{c r}=\left(\sum_{K \in \mathcal{T}}\left(\eta_{K}^{c r}\right)^{2}\right)^{1 / 2}=\left(\sum_{K \in \mathcal{T}}\left(\eta_{r, K}^{c r}\right)^{2}+\sum_{F \in \mathcal{E}_{I}}\left(\eta_{j, n, F}^{c r}\right)^{2}+\sum_{F \in \mathcal{E}}\left(\eta_{j, u, F}^{c r}\right)^{2}\right)^{1 / 2} .
$$


Lemma 3.3. Let $F \in \mathcal{E}_{I}$. For any $v^{c r} \in V^{c r}$, we have

$$
\left\|\llbracket v^{c r} \rrbracket\right\|_{0, F} \begin{cases}=\frac{1}{\sqrt{12}} h_{F}\left\|\llbracket \nabla v^{c r} \cdot \mathbf{t}_{F} \rrbracket\right\|_{0, F} & \text { if } d=2, \\ \leq h_{F}\left\|\llbracket \nabla v^{c r} \times \mathbf{n}_{F} \rrbracket\right\|_{0, F} & \text { if } d=3 .\end{cases}
$$

Proof. The equality in (3.10) is proved in [7] by a direct calculation. To show the validity of the inequality in (3.10), for any $v^{c r} \in V^{c r}$, without loss of generality, assume that the face $F$ lies in the $x y$ plane and that the centroid of $F$ is at the origin. Then there exist constants $a$ and $b$ such that

$$
\llbracket v^{c r} \rrbracket_{F}=a x+b y \quad \text { and } \quad \llbracket \nabla v^{c r} \times \mathbf{n}_{F} \rrbracket_{F}=(b,-a, 0) .
$$

Since $h_{F}$ is the diameter of the circumcircle of $F$, we have that $x^{2}+y^{2} \leq h_{F}^{2}$ for all $(x, y) \in F$. Now, it follows the Cauchy-Schwartz inequality that

$$
\left\|\llbracket v^{c r} \rrbracket\right\|_{0, F}^{2}=\iint_{F}(a x+b y)^{2} d x d y \leq\left(a^{2}+b^{2}\right) \iint_{F}\left(x^{2}+y^{2}\right) d x d y=h_{F}^{2}\left\|\llbracket \nabla v^{c r} \times \mathbf{n}_{F} \rrbracket\right\|_{0, F}^{2} .
$$

This completes the proof of the lemma.

Remark 3.4. Instead of the face solution jumps, existing residual based error estimators for the nonconforming elements use the face tangential derivative jumps $\eta_{j, t, F}^{c r}$. Lemma 3.3 indicates that

$$
\eta_{j, u, F}^{c r}<\eta_{j, t, F}^{c r} .
$$

Thus, our estimator $\eta_{c r}$ is less than the existing estimator and, hence, it is more accurate (see Figure 6 in [7]).

Without the QMA, the following robust local efficiency bound

$$
\eta_{K}^{c r} \leq C\left(\left\|\alpha^{1 / 2} \nabla\left(u-u^{c r}\right)\right\|_{\triangle_{K}}+\operatorname{osc}_{1, \alpha}\left(f, \triangle_{K}\right)\right)
$$

can be found in Theorem 6.8 of [10] and Theorem 5.1 of [7]. The key idea of the proof on (3.11) is to use either element or edge bubble functions in order to localize the error as well as to simplify the boundary conditions.

3.2.2. Reliability bound. Without the QMA, the robust reliability bound for the nonconforming elements was first established in [7] for a slight modification of the estimator $\eta_{c r}$ in two dimensions. The modification is due to the failure of bounding the solution jump term. This difficulty may be overcome by using the trace inequality introduced in section 2 .

THEOREM 3.5. Let $u$ and $u^{\text {cr }}$ be the solutions of (1.3) and (3.1), respectively. Without the QMA in both two and three dimensions, the estimator $\eta_{c r}$ for the nonconforming elements satisfies the following robust reliability bound:

$$
\left\|\alpha^{1 / 2} \nabla_{h}\left(u-u^{c r}\right)\right\|_{0} \leq C\left(\eta_{c r}+\operatorname{osc}_{1, \alpha}(f)\right),
$$

where $C$ is a positive constant independent of $\alpha$.

Proof. Let

$$
e^{c r}=u-u^{c r} \quad \text { and } \quad e_{I}^{c r}=I^{c r} u-u^{c r}=I^{c r} e^{c r} .
$$


Then we have the following $L^{2}$ representation of the true error in the (broken) energy norm (see Lemma 2.1 of [7]):

$$
\begin{aligned}
\left\|\alpha^{1 / 2} \nabla_{h} e^{c r}\right\|_{0}^{2}= & \sum_{K \in \mathcal{T}}\left(f, e^{c r}-e_{I}^{c r}\right)_{K}-\sum_{F \in \mathcal{E}_{I}} \int_{F} j_{n, F}^{c r}\left\{e^{c r}-e_{I}^{c r}\right\}^{w} d s \\
& -\sum_{F \in \mathcal{E}} \int_{F}\left\{\alpha \nabla e^{c r} \cdot \mathbf{n}\right\}_{w} \llbracket u^{c r} \rrbracket d s .
\end{aligned}
$$

The first two terms of the above equality may be bounded in a similar fashion as that in [7]. That is, it follows from the Cauchy-Schwarz and triangle or trace inequalities, (3.2), and (3.9) that

$$
\left(f, e^{c r}-e_{I}^{c r}\right)_{K} \leq C\left(\eta_{r, K}^{c r}+\operatorname{osc}_{1, \alpha}(f, K)\right)\left\|\alpha^{1 / 2} \nabla_{h} e^{c r}\right\|_{0, K} \quad \forall K \in \mathcal{T}
$$

and

$$
\begin{aligned}
\int_{F} j_{n, F}^{c r}\left\{e-e_{I}^{c r}\right\}^{w} d s & \leq C\left\|j_{n, F}^{c r}\right\|_{0, F}\left\{\omega^{+}\left\|\left.\left(e^{c r}-e_{I}^{c r}\right)\right|_{K_{F}^{-}}\right\|_{0, F}+\omega^{-}\left\|\left(e^{c r}-e_{I}^{c r}\right)_{K_{F}^{+}}\right\|_{0, F}\right\} \\
& \leq C \sqrt{\frac{h_{F}}{\alpha_{F, A}}}\left\|j_{n, F}^{c r}\right\|_{0, F}\left\|\alpha^{1 / 2} \nabla e^{c r}\right\|_{K_{F}^{+} \cup K_{F}^{-}} \quad \forall F \in \mathcal{E}_{I} .
\end{aligned}
$$

To bound the third term on the solution jump, the key is the inequality in (2.7), which together with (3.9) and the local efficiency bound of the element residual, yields

$$
\begin{aligned}
& \int_{F}\left\{\alpha \nabla e^{c r} \cdot \mathbf{n}_{F}\right\}_{w} \llbracket u^{c r} \rrbracket d s \\
& =\int_{F} \llbracket u^{c r} \rrbracket\left(\left.\omega^{+}\left(\alpha^{+} \nabla e^{c r} \cdot \mathbf{n}\right)\right|_{K^{+}}+\left.\omega^{-}\left(\alpha^{-} \nabla e^{c r} \cdot \mathbf{n}\right)\right|_{K^{-}}\right) d s \\
& \leq C \sqrt{\frac{\alpha_{F, H}}{h_{F}}}\left\|\llbracket u^{c r} \rrbracket\right\|_{0, F} \sum_{K \in \mathcal{T}_{F}}\left(\left\|\alpha^{1 / 2} \nabla e^{c r}\right\|_{0, K}+h_{K} \alpha_{K}^{-1 / 2}\left\|f+\nabla \cdot\left(\alpha \nabla u^{c r}\right)\right\|_{0, K}\right) \\
& \quad \leq C \eta_{j, u, F}^{c r} \sum_{K \in \mathcal{T}_{F}}\left(\left\|\alpha^{1 / 2} \nabla_{h} e^{c r}\right\|_{0, K}+\operatorname{osc}_{1, \alpha}(f, K)\right) \quad \forall F \in \mathcal{E} .
\end{aligned}
$$

Summing (3.13) over $K \in \mathcal{T}$, (3.14) over $F \in \mathcal{E}_{I}$, and (3.15) over $F \in \mathcal{E}$ implies the validity of (3.12). This completes the proof of the theorem.

4. Discontinuous Galerkin finite element method. This section establishes the a priori and the residual based a posteriori error estimates for the discontinuous Galerkin finite element approximation.

For $s>0$, let

$$
\begin{aligned}
H^{1+s}(\mathcal{T}) & =\left\{v \in L^{2}(\Omega):\left.v\right|_{K} \in H^{1+s}(K) \forall K \in \mathcal{T}\right\} \\
\text { and } V^{1+s}(\mathcal{T}) & =\left\{v \in H^{1+s}(K):\left.(\Delta v)\right|_{K} \in L^{2}(K) \forall K \in \mathcal{T}\right\} .
\end{aligned}
$$

In [8] we introduced the following variational formulation for the interface problem in (1.1) and (1.2): find $u \in V^{1+\epsilon}(\mathcal{T})$ with $\epsilon>0$ such that

$$
a_{d g}(u, v)=(f, v) \quad \forall v \in V^{1+\epsilon}(\mathcal{T}),
$$


where the bilinear form $a_{d g}(\cdot, \cdot)$ is given by

$$
\begin{aligned}
a_{d g}(u, v)= & \left(\alpha \nabla_{h} u, \nabla_{h} v\right)+\sum_{F \in \mathcal{E}} \int_{F} \gamma \frac{\alpha_{F}, H}{h_{F}} \llbracket u \rrbracket \llbracket v d s \\
& -\sum_{F \in \mathcal{E}} \int_{F}\left\{\alpha \nabla u \cdot \mathbf{n}_{F}\right\}_{w}^{F} \llbracket v \rrbracket d s-\sum_{F \in \mathcal{E}} \int_{F}\left\{\alpha \nabla v \cdot \mathbf{n}_{F}\right\}_{w}^{F} \llbracket u \rrbracket d s .
\end{aligned}
$$

The $\gamma$ is a positive constant only depending on the shape of elements.

Denote the discontinuous finite element space on the triangulation $\mathcal{T}$ by

$$
D_{k}=\left\{v \in L^{2}(\Omega):\left.v\right|_{K} \in P_{k}(K) \forall K \in \mathcal{T}\right\} ;
$$

the discontinuous Galerkin finite element method is then to seek $u_{k}^{d g} \in D_{k}$ such that

$$
a_{d g}\left(u_{k}^{d g}, v\right)=(f, v) \quad \forall v \in D_{k} .
$$

The difference between (4.1) and (4.2) leads to the following error equation:

$$
a_{d g}\left(u-u_{k}^{d g}, v\right)=0 \quad \forall v \in D_{k} .
$$

For simplicity, we consider only this symmetric version of the interior penalty discontinuous Galerkin finite element method since its extension to other versions of discontinuous Galerkin approximations is straightforward. Define the jump seminorm and the DG norm by

$$
\|v\|_{J, F}=\sqrt{\frac{\alpha_{F, H}}{h_{F}}}\|\llbracket v \rrbracket\|_{0, F} \quad \text { and } \quad\|v\|_{d g}=\left(\left\|\alpha^{1 / 2} \nabla_{h} v\right\|_{0, \Omega}^{2}+\sum_{F \in \mathcal{E}}\|v\|_{J, F}^{2}\right)^{1 / 2},
$$

respectively, for all $v \in H^{1}(\mathcal{T})$. It was shown in [8] that there exists a positive constant $C$ independent of the jump of $\alpha$ such that

$$
C\|v\|_{d g}^{2} \leq a_{d g}(v, v) \quad \forall v \in D_{k} .
$$

4.1. A priori error estimate. Let $f_{k}$ be the $L^{2}$ projection of $f$ onto $D_{k}$ for $k \geq 0$. Define local and global weighted oscillations by

$\operatorname{osc}_{k, \alpha}(f, K)=\frac{h_{K}}{\sqrt{\alpha_{K}}}\left\|f-f_{k-1}\right\|_{0, K} \quad$ and $\quad \operatorname{osc}_{k, \alpha}(f, \mathcal{T})=\left(\sum_{K \in \mathcal{T}} \operatorname{osc}_{k, \alpha}(f, K)^{2}\right)^{1 / 2}$,

respectively. To handle the case that local regularity $1+s_{K}$ of $u$ may be larger than 2 , we also use the following notation:

$$
\operatorname{app}_{\alpha}(f, K)= \begin{cases}\operatorname{osc}_{1, \alpha}(f, K) & \text { if } 0<s_{K}<1, \\ h_{K}^{\min \left\{k, s_{K}\right\}} \alpha_{K}^{1 / 2}|\nabla u|_{s_{K}, K} & \text { if } s_{K} \geq 1 .\end{cases}
$$

THEOREM 4.1. Let $u$ be the solution of (1.3) and $\left.u\right|_{K}$ be its restriction on $K \in \mathcal{T}$. Assume that $u \in H^{1+s}(\Omega) \cap V^{1+s}(\mathcal{T})$ with $s>0$ and that $\left.u\right|_{K} \in H^{1+s_{K}}(K)$ with elementwise defined $s_{K}>0$ for all $K \in \mathcal{T}$. Let $u_{k}^{d g} \in D_{k}$ be the discontinuous 
Galerkin finite element approximation in (4.2). In both two and three dimensions, we have the following error estimates:

$$
\begin{aligned}
\left\|u-u_{k}^{d g}\right\|_{d g} & \leq C\left(\inf _{v \in D_{k}}\|u-v\|_{d g}+\operatorname{osc}_{k, \alpha}(f, \mathcal{T})\right) \\
& \leq C\left(\sum_{K \in \mathcal{T}} h_{K}^{2 s_{K}}\left|\alpha^{1 / 2} \nabla u\right|_{s_{K}, K}^{2}+\operatorname{app}_{\alpha}(f, K)^{2}\right)^{1 / 2},
\end{aligned}
$$

where $C$ is a positive constant independent of the jump of the diffusion coefficient $\alpha$.

Proof. For any $F \in \mathcal{E}$, it follows from the trace inequality and (3.7) that for all $v \in D_{k}$

$$
\begin{aligned}
\sqrt{\alpha_{F, H} / h_{F}}\|\llbracket u-v \rrbracket\|_{0, F} & \leq \sqrt{\alpha_{F, H} / h_{F}}\left(\left\|\left.(u-v)\right|_{K_{F}^{+}}\right\|_{0, F}+\left\|\left.(u-v)\right|_{K_{F}^{-}}\right\|_{0, F}\right) \\
& \leq C \sum_{\kappa=-,+}\left(h_{K_{F}^{\kappa}}^{-1}\left\|\alpha^{1 / 2}(u-v)\right\|_{0, K_{F}^{\kappa}}+\left\|\alpha^{1 / 2} \nabla(u-v)\right\|_{0, K_{F}^{\kappa}}\right) .
\end{aligned}
$$

Since $\left.u\right|_{K} \in H^{1+s_{K}}(K)$ with $s_{K} \geq 1$, then $\left.f\right|_{K}=-\left.\alpha \Delta u\right|_{K} \in H^{s_{K}-1}(K)$. It is easy to show that

$$
\operatorname{osc}_{k, \alpha}(f, K) \leq h_{K}^{\min \left\{k, s_{K}\right\}} \alpha_{K}^{1 / 2}|\nabla u|_{s_{K}, K} .
$$

Now, the second inequality in (4.6) is a direct consequence of the first inequality in (4.6) and the elementwise approximation property of discontinuous piecewise polynomials. By the triangle inequality, we have

$$
\left\|u-u_{k}^{d g}\right\|_{d g} \leq\|u-v\|_{d g}+\left\|u_{k}^{d g}-v\right\|_{d g} \quad \forall v \in D_{k} .
$$

To show the validity of the first inequality in (4.6), it suffices to prove that

$$
\left\|u_{k}^{d g}-v\right\|_{d g} \leq C\left(\|u-v\|_{d g}+\operatorname{osc}_{k, \alpha}(f, \mathcal{T})\right) \quad \forall v \in D_{k} .
$$

To this end, for any $v \in D_{k}$, let

$$
e=u-v \quad \text { and } \quad e_{k}=u_{k}^{d g}-v .
$$

It follows from the coercivity in (4.4), the error equation in (4.3), the Cauchy-Schwarz inequality, the fact that $\llbracket u \rrbracket_{F}=0$ for all $F \in \mathcal{E}$, and the first inequality in (2.7) that

$$
\begin{aligned}
& C\left\|e_{k}\right\|_{d g}^{2} \leq a_{d g}\left(e_{k}, e_{k}\right)=a_{d g}\left(e, e_{k}\right) \\
& =\left(\alpha \nabla_{h} e, \nabla_{h} e_{k}\right)+\sum_{F \in \mathcal{E}} \int_{F} \frac{\gamma \alpha_{F}, H}{h_{F} \llbracket e \rrbracket \llbracket e_{k} \rrbracket} d s-\sum_{F \in \mathcal{E}} \int_{F}\left\{\alpha \nabla e_{k} \cdot \mathbf{n}\right\}_{w}^{F} \llbracket e \rrbracket d s \\
& \quad-\sum_{F \in \mathcal{E}} \int_{F}\{\alpha \nabla e \cdot \mathbf{n}\}_{w}^{F} \llbracket e_{k} \rrbracket d s \\
& \leq C\left\{\|e\|_{d g}\left\|e_{k}\right\|_{d g}+\sum_{F \in \mathcal{E}}\|\llbracket e \rrbracket\|_{0, F}\left\|\left\{\alpha \nabla e_{k} \cdot \mathbf{n}\right\}_{w}^{F}\right\|_{0, F}\right. \\
& \left.\quad+\sum_{F \in \mathcal{E}} h_{F}^{-1 / 2}\left\|\llbracket e_{k} \rrbracket\right\|_{0, F} \sum_{\kappa=-,+} w_{F}^{\kappa} \alpha_{F}^{\kappa}\left(\|\nabla e\|_{0, K_{F}^{\kappa}}+h_{K}\|\Delta e\|_{0, K_{F}^{\kappa}}\right)\right\} \\
& \equiv C\left(I_{1}+I_{2}+I_{3}\right) .
\end{aligned}
$$


By the triangle, trace, and inverse inequalities, we have that

$$
\left\|\left\{\alpha \nabla e_{k} \cdot \mathbf{n}\right\}_{w}^{F}\right\|_{0, F} \leq C \sum_{\kappa=-,+} w_{F}^{\kappa} h_{K_{F}^{\kappa}}^{-1 / 2} \alpha_{K_{F}^{\kappa}}^{1 / 2}\left\|\alpha^{1 / 2} \nabla e_{k}\right\|_{0, K_{F}^{\kappa}} .
$$

With the choice of the weights in (3.8), a simple calculation shows that

$$
w_{F}^{\kappa} \sqrt{\frac{\alpha_{F}^{\kappa}}{\alpha_{F, H}}} \leq \frac{\sqrt{2}}{2} \quad \text { for } \kappa=-,+.
$$

Together with the Cauchy-Schwarz inequality, we have

$$
\begin{aligned}
I_{2} & \leq C \sum_{F \in \mathcal{E}}\|\llbracket e \rrbracket\|_{J, F} \sum_{\kappa=-,+}\left\|\alpha^{1 / 2} \nabla e_{k}\right\|_{0, K_{F}^{\kappa}} \leq C\|e\|_{d g}\left\|e_{k}\right\|_{d g} \\
I_{3} & \leq C \sum_{F \in \mathcal{E}}\left\|\llbracket e_{k} \rrbracket\right\|_{J, F} \sum_{\kappa=-,+}\left(\left\|\alpha^{1 / 2} \nabla e\right\|_{0, K_{F}^{\kappa}}+h_{K_{F}^{\kappa}}\left\|\alpha^{1 / 2} \Delta e\right\|_{0, K_{F}^{\kappa}}\right) \\
& \leq C\left\|e_{k}\right\|_{d g}\left(\|e\|_{d g}+\left(\sum_{K \mathcal{T}} h_{K}^{2} \alpha_{K}\|\Delta e\|_{0, K}^{2}\right)^{1 / 2}\right)
\end{aligned}
$$

Combining those inequalities gives that

$$
\left\|e_{k}\right\|_{d g} \leq C\left(\|e\|_{d g}+\left(\sum_{K \in \mathcal{T}} h_{K}^{2} \alpha_{K}\|\Delta e\|_{0, K}^{2}\right)^{1 / 2}\right) .
$$

Now, (4.7) is a direct consequence of the following efficiency bound (see (4.8), or Lemma 5.2 in [8] for the linear case):

$$
h_{K} \alpha_{K}^{1 / 2}\|\Delta e\|_{0, K} \leq C\left(\left\|\alpha^{1 / 2} \nabla e\right\|_{0, \Delta_{K}}+\operatorname{osc}_{k, \alpha}\left(f, \Delta_{K}\right)\right),
$$

where $\Delta_{K}$ is a local patch of elements containing $K$. This completes the proof of the inequality in (4.7) and, hence, the theorem.

4.2. Residual-based a posteriori error estimator. This section describes the local indicator and global estimator for the discontinuous Galerkin finite element approximations and establishes the local efficiency and the global reliability bounds.

4.2.1. Error indicator and estimator. Denote the element residuals and the corresponding indicators by

$$
r_{K}^{d g}=f_{k-1}+\nabla \cdot\left(\alpha \nabla u_{k}^{d g}\right) \quad \text { and } \quad \eta_{r, K}^{d g}=\frac{h_{K}}{\sqrt{\alpha_{K}}}\left\|r_{K}^{d g}\right\|_{0, K} \quad \forall K \in \mathcal{T},
$$

respectively. Denote the respective face flux and solution jumps by

$$
j_{n, F}^{d g}=\llbracket \alpha \nabla_{h} u_{k}^{d g} \cdot \mathbf{n} \rrbracket_{F} \forall F \in \mathcal{E}_{I} \quad \text { and } \quad j_{u, F}^{d g}=\llbracket u_{k}^{d g} \rrbracket \forall F \in \mathcal{E}
$$

and the indicators corresponding to the face flux and solution jumps by

$$
\eta_{j, n, F}^{d g}=\sqrt{\frac{h_{F}}{\alpha_{F}, A}}\left\|j_{n, F}^{d g}\right\|_{0, F} \quad \text { and } \quad \eta_{j, u, F}^{d g}=\sqrt{\frac{\alpha_{F, H}}{h_{F}}}\left\|\llbracket u^{d g} \rrbracket\right\|_{0, F},
$$


respectively. Then the local indicator of the residual type for the discontinuous elements is given $[8]$ by

$$
\begin{aligned}
\eta_{K}^{d g}= & \left(\left(\eta_{r, K}^{d g}\right)^{2}+\sum_{F \in \mathcal{E}_{K} \cap \mathcal{E}_{I}} \frac{1}{2}\left(\eta_{j, n, F}^{d g}\right)^{2}+\sum_{F \in \mathcal{E}_{K} \cap \mathcal{E}_{I}} \frac{1}{2}\left(\eta_{j, u, F}^{d g}\right)^{2}\right. \\
& \left.+\sum_{F \in \mathcal{E}_{K} \cap \mathcal{E}_{D}}\left(\eta_{j, u, F}^{d g}\right)^{2}\right)^{1 / 2} .
\end{aligned}
$$

Now the global estimator for the discontinuous elements is given by

$$
\eta_{d g}=\left(\sum_{K \in \mathcal{T}}\left(\eta_{K}^{d g}\right)^{2}\right)^{1 / 2}=\left(\sum_{K \in \mathcal{T}}\left(\eta_{r, K}^{d g}\right)^{2}+\sum_{F \in \mathcal{E}_{I}}\left(\eta_{j, n, F}^{d g}\right)^{2}+\sum_{F \in \mathcal{E}}\left(\eta_{j, u, F}^{d g}\right)^{2}\right)^{1 / 2}
$$

The local efficiency bound of $\eta_{K}^{d g}$ for all $K \in \mathcal{T}$ was established without the QMA. For example, it is proved in [8, Lemma 5.2 and Theorem 5.3] for the linear approximation that for any $K \in \mathcal{T}$, there exists a positive constant $C$ independent of $\alpha$ and $h_{K}$ such that

$$
\eta_{K}^{d g} \leq C\left(\left\|u-u_{d g}^{k}\right\|_{\triangle_{K}}+\operatorname{osc}_{k, \alpha}\left(f, \triangle_{K}\right)\right),
$$

where $\triangle_{K}$ is a local neighborhood of $K$. The generalization to the higher degree approximation is trivial.

4.2.2. Reliability bound. Without the QMA, the robust reliability bound for the discontinuous Galerkin finite element method may be obtained in a similar fashion as that for the nonconforming elements. Again, the key steps are the $L^{2}$ representation of the true error and the inequality (2.7) to bound the solution jump. Moreover, we simply use the local constant average of the error instead of the modified Clément interpolation due to the complete local feature of the discontinuous elements.

Let $u$ and $u_{k}^{d g}$ be the solutions of (1.3) and (4.2), respectively. Denote the true error by

$$
e^{d g}=u-u_{k}^{d g} .
$$

Let $\bar{e}^{d g}$ be piecewise constant on $\mathcal{T}$ with $\left.\bar{e}^{d g}\right|_{K}$ being the average of $e^{d g}$ on $K \in \mathcal{T}$. It is well known that

$$
\left\|e^{d g}-\bar{e}^{d g}\right\|_{0, K} \leq C h_{K}\left\|\nabla e^{d g}\right\|_{0, K} \quad \forall K \in \mathcal{T},
$$

where $C$ only depends on the regularity of $\mathcal{T}$.

Lemma 4.2. The true error of the discontinuous finite element approximation in the broken energy norm has the following error representation:

$$
\begin{aligned}
\left\|\alpha^{1 / 2} \nabla_{h} e^{d g}\right\|_{0}^{2} & =\sum_{K \in \mathcal{T}}\left(f+\nabla \cdot\left(\alpha \nabla u_{k}^{d g}\right), e^{d g}-\bar{e}^{d g}\right)_{K}-\sum_{F \in \mathcal{E}} \int_{F}\left\{\alpha \nabla e^{d g} \cdot \mathbf{n}\right\}_{w} \llbracket u_{k}^{d g} \rrbracket d s \\
& -\sum_{F \in \mathcal{E}_{I}} \int_{F} \llbracket \alpha \nabla u^{d g} \cdot \mathbf{n} \rrbracket\left\{e^{d g}-\bar{e}^{d g}\right\}^{w} d s-\sum_{F \in \mathcal{E}} \int_{F} \gamma \frac{\alpha_{F}, H}{h_{F}} \llbracket u_{k}^{d g} \rrbracket \llbracket \bar{e}^{d g} \rrbracket d s .
\end{aligned}
$$


Proof. It follows from the error equation in (4.3), integrations by parts, (3.6), the continuities of the solution $u$ and the normal component of the flux $-\alpha \nabla u$ across any face $F \in \mathcal{E}_{I}$, and the homogeneous Dirichlet boundary condition that

$$
\begin{aligned}
& \left\|\alpha^{1 / 2} \nabla_{h} e^{d g}\right\|_{0}^{2}=\left(\alpha \nabla_{h} e^{d g}, \nabla_{h}\left(e^{d g}-\bar{e}^{d g}\right)\right) \\
= & \sum_{K \in \mathcal{T}}\left(f+\nabla \cdot\left(\alpha \nabla u_{k}^{d g}\right), e^{d g}-\bar{e}^{d g}\right)_{K}+\int_{\partial K}\left(\alpha \nabla e^{d g} \cdot \mathbf{n}\right)\left(e^{d g}-\bar{e}^{d g}\right) d s \\
= & \sum_{K \in \mathcal{T}}\left(f+\nabla \cdot\left(\alpha \nabla u_{k}^{d g}\right), e^{d g}-\bar{e}^{d g}\right)_{K}-\sum_{F \in \mathcal{E}_{I}} \int_{F}\left\{\alpha \nabla e^{d g} \cdot \mathbf{n}\right\}_{w} \llbracket u_{k}^{d g}+\bar{e}^{d g} \rrbracket d s \\
& -\sum_{F \in \mathcal{E}_{I}} \int_{F} \llbracket \alpha \nabla u^{d g} \cdot \mathbf{n} \rrbracket\left\{e^{d g}-\bar{e}^{d g}\right\}^{w} d s-\sum_{F \in \mathcal{E}_{D}} \int_{F}\left(\alpha \nabla e^{d g} \cdot \mathbf{n}\right)\left(u_{k}^{d g}+\bar{e}^{d g}\right) d s .
\end{aligned}
$$

On the other hand, the fact that $a_{d g}\left(e_{k}^{d g}, \bar{e}^{d g}\right)=0$ implies

$$
\begin{aligned}
\sum_{F \in \mathcal{E}} \int_{F} \gamma \frac{\alpha_{F}, H}{h_{F}} \llbracket e^{d g} \rrbracket \llbracket \bar{e}^{d g} \rrbracket d s & -\sum_{F \in \mathcal{E}_{I}} \int_{F}\left\{\alpha \nabla e^{d g} \cdot \mathbf{n}\right\}_{w} \llbracket \bar{e}^{d g} \rrbracket d s \\
& -\sum_{F \in \mathcal{E}_{D}} \int_{F}\left(\alpha \nabla e^{d g} \cdot \mathbf{n}\right) \bar{e}^{d g} d s=0 .
\end{aligned}
$$

Combining the above two equalities gives (4.10). This completes the proof of the lemma.

THEOREM 4.3. Let $u$ and $u_{k}^{d g}$ be the solution of (1.3) and (4.2), respectively. Without the QMA in both two and three dimensions, the estimator $\eta_{d g}$ for the discontinuous element approximation satisfies the following robust reliability bound:

$$
\left\|u-u_{k}^{d g}\right\|_{d g} \leq C\left(\eta_{d g}+\operatorname{osc}_{k, \alpha}(f, \mathcal{T})\right)
$$

where $C$ is a positive constant independent of $\alpha$.

Proof. By the definition of the DG norm $\|\cdot\|_{d g}$, to prove the validity of (4.11), it suffices to show that

$$
\left\|\alpha^{1 / 2} \nabla_{h}\left(u-u_{k}^{d g}\right)\right\|_{0} \leq C\left(\eta_{d g}+\operatorname{osc}_{k, \alpha}(f, \mathcal{T})\right) .
$$

To this end, denote the estimators corresponding to the element residual, the face flux jump, and the solution jump by

$$
\begin{aligned}
\eta_{r}^{d g}= & \left(\sum_{K \in \mathcal{T}}\left(\eta_{r, K}^{d g}\right)^{2}\right)^{1 / 2}, \quad \eta_{j, n}^{d g}=\left(\sum_{F \in \mathcal{E}_{I}}\left(\eta_{j, n, F}^{d g}\right)^{2}\right)^{1 / 2}, \\
& \text { and } \quad \eta_{j, u}^{d g}=\left(\sum_{F \in \mathcal{E}}\left(\eta_{j, u, F}^{d g}\right)^{2}\right)^{1 / 2},
\end{aligned}
$$

respectively. Denote four terms in Lemma 4.2 by $I_{1}, I_{2}, I_{3}$, and $I_{4}$, respectively. Hence,

$$
\left\|\alpha^{1 / 2} \nabla_{h}\left(u-u_{k}^{d g}\right)\right\|_{0}^{2}=I_{1}+I_{2}+I_{3}+I_{4} .
$$


In a similar fashion as the proof of Theorem 3.5, the $I_{i}$ for $i=1,2,3$ may be bounded as follows:

$$
\begin{aligned}
& I_{1} \leq C\left(\eta_{r}^{d g}+\operatorname{osc}_{k, \alpha}(f, \mathcal{T})\right)\left\|\alpha^{1 / 2} \nabla_{h} e^{d g}\right\|_{0} \\
& I_{2} \leq C \eta_{j, u}^{d g}\left(\left\|\alpha^{1 / 2} \nabla_{h} e^{d g}\right\|_{0}+\operatorname{osc}_{k, \alpha}(f, \mathcal{T})\right)
\end{aligned}
$$

and $\quad I_{3} \leq C \eta_{j, n}^{d g}\left\|\alpha^{1 / 2} \nabla_{h} e^{d g}\right\|_{0}$.

To bound $I_{4}$, for all $F \in \mathcal{E}$, it follows from the triangle and the Cauchy-Schwartz inequalities, the continuity of the solution $u$, the trace inequality, and (4.9) that

$$
\begin{aligned}
& \int_{F} \frac{\alpha_{F, H}}{h_{F}} \llbracket u_{k}^{d g} \rrbracket \llbracket \bar{e}^{d g} \rrbracket d s \leq \int_{F} \frac{\alpha_{F, H}}{h_{F}} \llbracket u_{k}^{d g} \rrbracket\left(\left\|\llbracket e^{d g} \rrbracket\right\|_{0, F}+\left\|\llbracket e^{d g}-\bar{e}^{d g} \rrbracket\right\|_{0, F}\right) d s \\
\leq & \left(\eta_{j, u, F}^{d g}\right)^{2}+\eta_{j, u, F}^{d g} \sqrt{\frac{\alpha_{F, H}}{h_{F}}}\left\|\llbracket e^{d g}-\bar{e}^{d g} \rrbracket\right\|_{0, F} \leq\left(\eta_{j, u, F}^{d g}\right)^{2} \\
& +C \eta_{j, u, F}^{d g} \sum_{K \in \mathcal{T}_{F}}\left\|\alpha^{1 / 2} \nabla_{h} e^{d g}\right\|_{0, K} .
\end{aligned}
$$

Summing over all faces $F \in \mathcal{E}$ and using the Cauchy-Schwarz inequality give

$$
I_{4}=\gamma \sum_{F \in \mathcal{E}} \int_{F} \frac{\alpha_{F, H}}{h_{F}} \llbracket u_{k}^{d g} \rrbracket \llbracket \bar{e}^{d g} \rrbracket d s \leq C\left(\left(\eta_{j, u}^{d g}\right)^{2}+\eta_{j, u}^{d g}\left\|\alpha^{1 / 2} \nabla_{h} e^{d g}\right\|_{0}\right) .
$$

Combining the bounds for all $I_{i}$ and using the Cauchy-Schwarz inequality, we have

$$
\begin{aligned}
\left\|\alpha^{1 / 2} \nabla_{h} e^{d g}\right\|_{0}^{2} \leq & C\left(\eta_{r}^{d g}+\eta_{j, n}^{d g}+\eta_{j, u}^{d g}+\operatorname{osc}_{k, \alpha}(f, \mathcal{T})\right)\left\|\alpha^{1 / 2} \nabla_{h} e^{d g}\right\|_{0} \\
& +C\left(\left(\eta_{j, u}^{d g}\right)^{2}+\operatorname{osc}_{k, \alpha}(f, \mathcal{T})^{2}\right)
\end{aligned}
$$

which, together with the inequality $2 a b \leq a^{2}+b^{2}$, implies

$$
\left\|\alpha^{1 / 2} \nabla_{h} e^{d g}\right\|_{0} \leq C\left(\eta_{d g}+\operatorname{osc}_{k, \alpha}(f, \mathcal{T})\right) .
$$

This proves the validity of (4.12) and, hence, the theorem. This completes the proof of the theorem.

\section{REFERENCES}

[1] M. Ainsworth, Robust a posteriori error estimation for nonconforming finite element approximation, SIAM J. Numer. Anal., 42 (2005), pp. 2320-2341.

[2] M. Ainsworth, A posteriori error estimation for lowest order Raviart-Thomas mixed finite elements, SIAM J. Sci. Comput., 30 (2007), pp. 189-204.

[3] D. N. Arnold, F. Brezzi, B. Cockburn, and L. D. Marini, Unified analysis of discontinuous Galerkin methods for elliptic problems, SIAM J. Numer. Anal., 39 (2002), pp. 1749-1779.

[4] C. Bernardi And F. Hecht, Error indicators for the mortar finite element discretization of the Laplace equation, Math. Comp., 71 (2001), pp. 1371-1403.

[5] C. Bernardi and R. Verfürth, Adaptive finite element methods for elliptic equations with non-smooth coefficients, Numer. Math., 85 (2000), pp. 579-608.

[6] D. Boffi, F. Brezzi, And M. Fortin, Mixed Finite Element Methods and Applications, Springer Ser. Comput. Math. 44, Spinger, New York, 2013.

[7] Z. CAI, C. HE, AND S. ZHANG, Residual-based a posteriori error estimate for interface problems: Nonconforming linear elements, Math. Comp. (2016). 
[8] Z. CAI, X. Ye, AND S. Zhang, Discontinuous Galerkin finite element methods for interface problems: A priori and a posteriori error estimations, SIAM J. Numer. Anal., 49 (2011), pp. 1761-1787.

[9] Z. CAi And S. Zhang, Recovery-based error estimator for interface problems: Conforming linear elements, SIAM J. Numer. Anal., 47 (2009), pp. 2132-2156.

[10] Z. CAI AND S. Zhang, Recovery-based error estimator for interface problems: Mixed and nonconforming elements, SIAM J. Numer. Anal., 48 (2010), pp. 30-52.

[11] Z. CAI AND S. ZHANG, Robust equilibrated residual error estimator for diffusion problems: Conforming elements, SIAM J. Numer. Anal., 50 (2012), pp. 151-170.

[12] Z. Cai And S. Zhang, Finite Element Methods for Interface Problems: Robust Comparisons Results, manuscript, 2015.

[13] P. G. Ciarlet, The Finite Element Method for Elliptic Problems, SIAM Classics in Appl. Math. 40, SIAM, Philadelphia, 2002.

[14] S. Du, R. Lin, AND Z. Zhang, A posteriori error analysis of multipoint flux mixed finite element method for interface problems, Adv. Comput. Math., 42 (2016), pp. 921-945.

[15] M. Dryja, M. V. Sarkis, and O. B. Widlund, Multilevel Schwartz method for elliptic problems with discontinuous coefficients in three dimensions, Numer. Math., 72 (1996), pp. 313-348.

[16] T. Dupont and R. Scott, Polynomial approximation of functions in Sobolev spaces, Math. Comp., 34 (1980), pp. 441-463.

[17] A. Ern and J.-L. Guermond, Theory and Practice of Finite Elements, Appl. Math. Ser. 159, Springer, New York, 2004.

[18] A. ERn And A. Stephansen, A posteriori energy-norm error estimates for discontinuous Galerkin method with weighted averages for advection-diffusion equations approximated by weighted interiori penalty methods, J. Comput. Math., 26 (2008), pp. 488-510.

[19] A. Ern, A. Stephansen, And P. Zunino, A discontinuous Galerkin method with weighted averages for advection-diffusion equations with locally small and anisotropic diffusivity, IMA J. Numer. Anal., 29 (2009), pp. 235-256.

[20] P. Grisvard, Elliptic Problems in Nonsmooth Domains, Monogr. Stud. Math. 24, Pitman, Boston, 1985.

[21] T. GudI, A new error analysis for discontinuous finite element methods for linear elliptic problems, Math. Comp., 79 (2010), pp. 2169-2189.

[22] R. B. KellogG, On the Poisson equation with intersecting interfaces, Appl. Anal., 4 (1975), pp. 101-129.

[23] K-Y. Kiм, A posteriori error analysis for locally conservative mixed methods, Math. Comp., 76 (2007), pp. 43-66.

[24] R. Luce And B. I. Wohlmuth, A local a posteriori error estimator based on equilibrated fluxes, SIAM J. Numer. Anal., 42 (2004), pp. 1394-1414.

[25] W. MCLEAn, Strongly Elliptic Systems and Boundary Integral Equations, Cambridge University Press, Cambridge, UK, 2000.

[26] R. H. Nochetto And A. Veeser, Primer of adaptive finite element methods, in Multiscale and Adaptivity: Modeling, Numerics and Applications, S. Bertoluzza, R. H. Nochetto, A. Quarteroni, K. G. Siebert, and A. Veeser, eds., Lecture Notes in Math. 2040, Springer, 2012, pp. 125-225

[27] M. Petzoldt, Regularity and Error Estimators for Elliptic Problems with Discontinuous Coefficients, Ph.D. thesis, Freien Universitat Berlin, 2001.

[28] M. Petzoldt, A posteriori error estimators for elliptic equations with discontinuous coeffcients, Adv. Comput. Math., 16 (2002), pp. 47-75.

[29] P. A. Raviart and J. M. Thomas, A mixed finite element method for second order elliptic problems, in Mathematical Aspects of the Finite Element Method, I. Galligani and E. Magenes, eds., Lecture Notes in Math. 606, Springer, Berlin, 1977.

[30] B. RiviÈRe, Discontinuous Galerkin Methods for Solving Elliptic and Parabolic Equations: Theory and Implementation, SIAM, Philadelphia, 2008.

[31] M. Vohralk, Guaranteed and fully robust a posteriori error estimates for conforming discretizations of diffusion problems with discontinuous coefficients, J. Sci. Comput., 46 (2011), pp. 397-438. 